\title{
Cultura y valores de la sociedad civil: las entidades de voluntariado
}

\author{
Juan Sebastián Fernández Prados \\ Universidad de Almería
}

\begin{abstract}
RESUMEN
El resurgimiento del concepto de sociedad civil en las ciencias sociales tiene una de sus manifestaciones en el aumento de las entidades de voluntariado en las últimas décadas. Este resurgimiento y aumento de la participación asociada de la ciudadanía está siendo, en términos de R. Inglehart, uno de los indicadores del cambio cultural hacia una sociedad de valores cada vez más postmaterialistas. En este trabajo se pretende delimitar el concepto de sociedad civil siguiendo el modelo aportado por J. L. Cohen y A. Arato, y ubicar dentro de éste las entidades de voluntariado y organizaciones del Tercer Sector. Además, se persigue demostrar, por un lado, que los miembros activos en organizaciones caritativas y sociales son parte de la punta de lanza del cambio de valores, y mostrar, por otro lado, los rasgos principales de la cultura política y social del voluntariado.
\end{abstract}

Palabras clave: Sociedad Civil, Cambio Cultural, Voluntariado, Valores Sociales. 
Retorno, emergencia, revitalización, resurrección, resurgimiento, renacimiento, reconstrucción, consolidación, primacía, auge, valor en alza... aparecen como términos que, en distintos ámbitos, acompañan al concepto de sociedad civil, subrayando unos la vuelta a un papel destacado perdido en el pasado, señalando otros un espacio novedoso y claramente distinto del Estado, enfatizando todos la puesta en escena de esta idea de un modo más patente en el debate público.

Antes de dilucidar las distintas aproximaciones teóricas y conceptuales a la sociedad civil, es preciso considerar cuáles son las razones más importantes que vuelven a ponerla en la palestra y en el candelero. ¿Por qué se vuelve a hablar de sociedad civil? En síntesis, destacan: la realidad de la práctica social y política, las crisis y transformaciones de los modelos sociales y, por último, el rescate del término en los discursos intelectuales.

Estos tres motivos nos pueden hacer pensar que no es una moda, ya que se constata un movimiento real y global por la infinidad de experiencias en la práctica totalidad del mundo ${ }^{1}$; crítico por ser factor principal o coadyuvante en muchas ocasiones de las transformaciones en los modelos y sistemas sociales vigentes ${ }^{2}$; y, al final, teórico por el carácter reflexivo y comprometido que supone para muchos intelectuales contemporáneos el replanteamiento de un concepto y una realidad social y política como es la sociedad civil ${ }^{3}$.

\footnotetext{
1 Ulrich Beck califica a la sociedad civil actual de transnacional o global, por haber adquirido en el proceso de globalización unas dimensiones hasta hoy desconocidas a nivel mundial; que implica, por una parte, dejar atrás el monopolio de los Estados nacionales en la política internacional y, por otra, puede suponer el mejor antídoto contra el globalismo de las transnacionales económicas. Estas organizaciones internacionales, en las que incluye las organizaciones no gubernamentales, configuran una red imbricada sinérgicamente con otros elementos y, cada vez más densa, de participación ciudadana cargada de futuro; que conlleva una fuerte crítica a los planteamientos pesimistas de ciertos postmodernos y a la ideología neoliberal del globalismo o reduccionismo economicista (Beck, 1998). El calificativo de mundial lo asocian Miguel Darcy y Rajesh Tandon a la sociedad civil porque durante las últimas dos décadas han detectado un crecimiento en la organización ciudadana en todos los continentes, relatando multitud de ejemplos que muestran la coordinación internacional de la acción cívica (Darcy de Oliveira y Tandon, 1996).
}

2 Arato destaca el papel jugado por la sociedad civil en la transición hacia la democracia de los países del antiguo bloque soviético (Arato, 2000).

\footnotetext{
3 No faltan los autores que opinan lo contrario, como es el caso de Robert D. Putnam. Afirma que el «Capital social» de Estados Unidos está en franca decadencia o en deterioro. La vitalidad de la sociedad civil ha declinado en las últimas décadas, tal y como se constata en la bajada de participación en la urnas, en reuniones políticas, falta de confianza en el Gobierno y en el fuerte proceso de individualización y privatización del elemento que ha asociado tradicionalmente a los estadounidenses: la religión (Putnam, 1996). $U$ otros que directamente apuntan y cuestionan las organizaciones de la sociedad civil, como las organizaciones no gubernamentales: por haber fracasado en su intento de ocuparse de los problemas que el sector privado o el Estado han tratado tradicionalmente; por su dependencia y condicionamiento de las donaciones; por su concepción de la participación como mera asunción de los objetivos definidos previamente; por no ser realmente competitivos en la ayuda comparativamente con el Estado, y por no respetar su criterio de llegar a los más débiles o pobres, etc. Estas apreciaciones del papel de las ONG, que aparecieron como un medio para fomentar la participación y la democracia mejorando la sociedad civil, llevan a la conclusión del fracaso de estas organizaciones y la necesidad de regresar al Estado. Las evidencias provenientes de Estados Unidos muestran que, de hecho, las ONG parecen durar sólo mientras las instituciones del Estado y del Mercado se muestran inadecuadas (Zaidi, 1998: 84). Y, finalmente, aquellos que ubican a las ONG fuera de los movimientos sociales, como simples grupos de presión en tanto en cuanto incorporan su acción política a los sistemas democráticos, abandonando su lógica participativa y su demanda de cambio social (Revilla Blanco, 2002: 18).
} 


\section{LA SOCIEDAD CIVIL: EL ESPACIO PARA UBICAR LAS ENTIDADES DE VOLUNTARIADO}

Las distintas propuestas que han aparecido en el debate contemporáneo sobre los límites de la sociedad civil se pueden sistematizar siguiendo el esquema propuesto por Víctor Pérez-Díaz ${ }^{4}$, que diferencia tres versiones representadas visualmente en el gráfico 1. La caracterización de cada una de ellas parte del modelo que divide la sociedad en tres grandes sectores (económico, político y social) a los cuales vienen asociados, respectivamente, instituciones sociopolíticas, socioeconómicas y movimientos sociales o asociaciones; además, lo completa la esfera pública o lugar de debate y participación en las sociedades contemporáneas. La exclusión o inclusión de los distintos componentes institucionales en la sociedad civil ofrece como resultado tres versiones o modelos diferenciados en su concepción y límites: amplia, reducida y restringida:

a) Versión amplia (modelo generalista): Incluye en la sociedad civil todas las instituciones de carácter social, político y económico que se generan en la esfera pública, en el Mercado o como fruto del imperio de la ley y de la existencia de la autoridad (Gellner, 1996, 1998; Pérez-Díaz, 1996, 1997).

b) Versión reducida (modelo dicotómico): Incluye en la sociedad civil sólo a aquellos actores sociales no gubernamentales, es decir, tanto los elementos del mercado económico como las asociaciones y organizaciones sociales que participan en la esfera pública (Keane, 1988, 1992; Giner, 1996, 1998).

c) Versión restringida (modelo de las tres partes o sectores): Excluye todos aquellos elementos gubernamentales y del mercado económico, reduciendo la sociedad civil al tejido asociativo de carácter voluntario y a los movimientos sociales (Arato, 1996; Cortina, 1994) ${ }^{5}$.

La cuestión sobre los límites y contenidos institucionales de la sociedad civil no representa una pregunta baladí, dado que nos ayudará a saber de lo que estamos hablando realmente, y a desvelar, en algunos casos, detrás de una definición y análisis determinado, la propuesta ideológica y política sobre las funciones que debe cumplir, el significado e importancia que posee y el proyecto sociopolítico que deseamos diseñar desde esta categoría

\footnotetext{
4 Pérez-Díaz, 1994: 13; 1997: 62.

5 Sin embargo, sus posturas parecen haber variado últimamente, ya que en sus últimas publicaciones dedicadas a la sociedad civil concluye, tras una crítica demoledora a Habermas y a su concepción restringida, que la sociedad civil abarca no sólo las asociaciones voluntarias y la esfera de la opinión pública, sino también las organizaciones e instituciones del mundo económico (Cortina, 1998: 379).
} 
GRÁFICO 1

Versiones sobre los límites de la sociedad civil

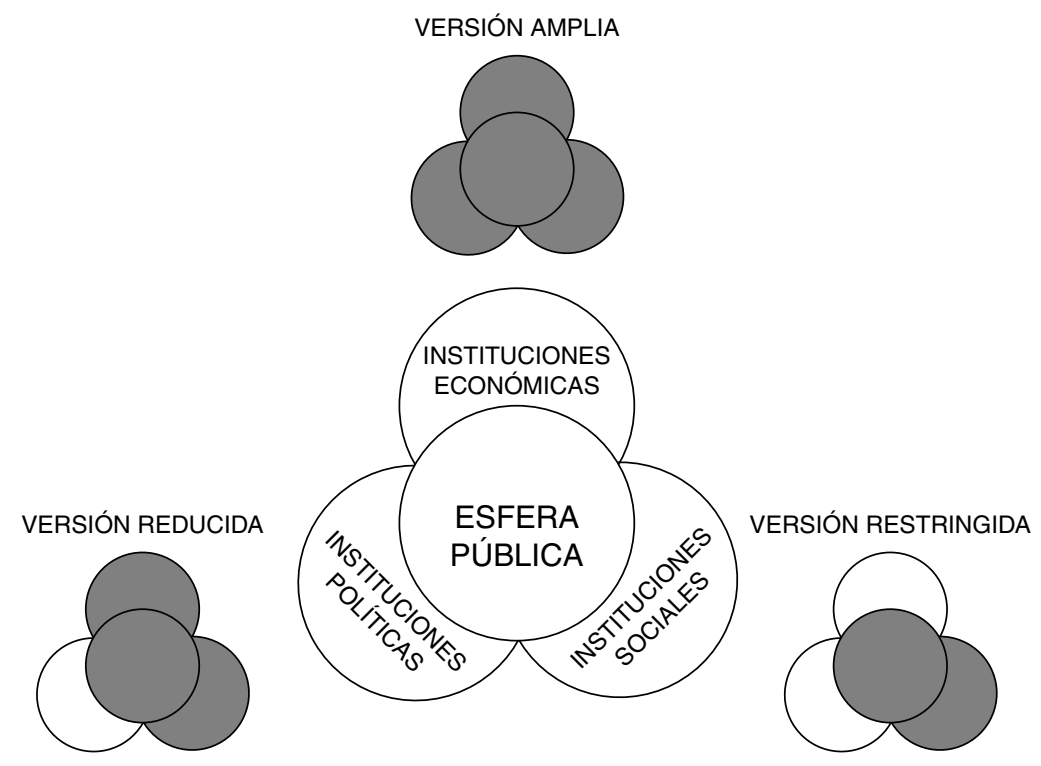

FUENTE:

Elaboración propia.

social ${ }^{6}$. De ahí se infiere que el uso, aplicación y designación de términos de orden partidista o provenientes de determinadas ideologías (liberal, neoliberal, marxista, socialista, revisionista...) sean frecuentes en la aclaración y delimitación de un concepto, obligadamente, político. En definitiva, la sociedad civil surge como el concepto ineludible para situar determinados fenómenos e iniciativas sociales en su espacio o esfera más adecuado, y para comprender los valores y la cultura de los mismos.

Las críticas vertidas a cada uno de los modelos (generalista, dicotómico y tripartito) se han construido muchas de ellas desde las censuras teóricas e ideológicas de alguno de sus oponentes, como es el caso de Víctor Pérez-Díaz y sus objeciones a la concepción haber-

6 Jeffrey C. Alexander es aún más contundente: «Introduciré un modelo analítico de relaciones entre la sociedad civil y los otros tipos de esferas institucionales que componen la sociedad. Sugeriré que, únicamente a través de las esferas sociales, podemos convertir la sociedad civil desde un concepto normativo a uno "real" que puede estudiarse de una manera científicosocial» (Alexander, 1998: 3) [traducción propia]. 
masiana y a toda la tradición marxista-revolucionaria; Cohen y Arato y sus argumentaciones en contra del modelo dicotómico marxista o liberal; Giner, opuesto a la concepción tripartita y a todas las falacias que genera, etc. (Giner y Sarasa, 1995: 70).

A pesar de las diferencias señaladas entre las distintas propuestas que se han presentado, podemos constatar algunas coincidencias, más de orden general y circunstancial que sustancial. El empleo de la indagación histórica para justificar sus posturas se hace reiterativo en todos los autores mencionados, ya sea planteada como una secuencia lineal o como el debate dialéctico entre al menos dos tradiciones. Otro punto de encuentro es la constatación de que estamos viviendo un reflujo o re-emergencia de la sociedad civil que la ha devuelto al terreno del debate en el pensamiento político y que, por ello, urge una reflexión para comprenderla y circunscribirla a su verdadera dimensión.

El argumento iusnaturalista ha desaparecido de todos los planteamientos que hemos esbozado, lo cual sugiere la toma de conciencia de que determinadas discusiones son estériles e innecesarias para entender un fenómeno eminentemente social y político. Por último, el recurso a esquemas y modelos más complejos o la incorporación de otros conceptos para completar el análisis institucional de la sociedad civil es recurrente, como, por ejemplo, privado-público, mundo de la vida-sistema, y sobre todo con la democracia y sus componentes (Diamond, 2001).

La opción por uno de estos modelos de sociedad civil se considera vital para la posterior comprensión del fenómeno o aspecto social que se desea estudiar (las entidades de voluntariado, en nuestro caso), porque va a servir de marco teórico explicativo y reforzador de las conclusiones de nuestro trabajo. La elección que hemos hecho tras este sucinto recorrido contemporáneo por las distintas versiones y modelos de sociedad civil es por el tercero que representa el defendido por Cohen y Arato, y que configuran una concepción tripartita de la sociedad y restringida de la sociedad civil.

A pesar de las diatribas lanzadas en contra de las tesis de los autores americanos con influencias habermasianas, la opción por esta posición es ventajosa por tres razones: la primera, porque aciertan al optar por una versión restringida de la sociedad civil, con el marco adecuado para discernir toda la amalgama de iniciativas sociales (ONG, entidades de voluntariado y organizaciones del Tercer Sector) y diferenciarla claramente de las otras que parten de los otros dos reinos o sectores, el político y el económico; la segunda, porque enmarcan adecuadamente a la sociedad civil asociándola con el Mundo de la Vida, en el sentido que le confiere Habermas, como el escenario extenso desde el cual interpretar las interacciones comunicativas, integradoras y solidarias de ésta; y, por último, porque expresan, en su proyección política y utópica, la esperanza 
emancipatoria y liberadora de la sociedad civil, apoyándose y compatibilizándose con una revolución democrática y con un Estado de Bienestar (Cohen y Arato, 1994: 423; Cohen, 1998: 36).

\section{EL CAMBIO CULTURAL HACIA UNA SOCIEDAD POSTMATERIALISTA: LA TENDENCIA PARA COMPRENDER LAS ENTIDADES DE VOLUNTARIADO}

Ciertamente, el protagonismo alcanzado por el concepto de sociedad civil está vinculado íntimamente con las transformaciones sociales e históricas acontecidas en las últimas décadas; la aproximación desde las ciencias sociales a la categoría sociológica del cambio social se torna imprescindible para comprender las organizaciones civiles y de voluntariado, y su resurgimiento de la mano de la sociedad civil. El término de cambio social aparece estrechamente vinculado a la sociología de tres modos distintos. En primer lugar, son las transformaciones sociales que sucedieron a lo largo de los siglos XVIII y XIX el marco histórico donde la sociología establece sus orígenes. En segundo lugar, es el cambio social como tal el que se convierte en el objeto de estudio de la sociología, ya sea para explicarlo y comprenderlo o para orientar, transformar y prever los acontecimientos futuros de la sociedad. Y, por último, el cambio se está convirtiendo en un factor crítico para la sociología, no sólo porque haya dejado en evidencia más de una vez a las teorías de esta reciente ciencia de lo social, sino porque cuestiona radicalmente los fundamentos epistemológicos de una fusión entre el positivismo científico y la compleja e imprevisible realidad de lo social.

La cuestión actual sobre el cambio social gira en torno a la pregunta sobre si la modernidad ha concluido o no; y es en este punto donde comienzan las diferencias entre dos grandes corrientes de pensamiento contemporáneo. Para unos, los postmodernos, la modernidad ha llegado a su fin; para los otros, los modernos reflexivos, el proyecto ilustrado se encuentra en plena expansión y afianzamiento. Los sociólogos y pensadores más destacados situados en esta última perspectiva son fundamentalmente, por una parte, J. Habermas, que ha censurado a los postmodernos por su neoconservadurismo y ha subrayado que el proyecto de la modernidad todavía no ha llegado a su conclusión, y, por otra, un conjunto de científicos sociales, entre los que destacan Giddens y Beck, que niegan el cambio social revolucionario propugnado por los postmodernos, apostando por apuntar una nueva etapa de la modernidad que denominan como reflexiva.

Las cuestiones abiertas, por tanto, en este debate serían básicamente tres:

- ¿Existe realmente un cambio radical o no en el orden social y cultural? 
- ¿Cuál es la vinculación entre los cambios sociales y culturales, es decir, entre el desarrollo económico y las transformaciones en el mundo de las ideas y los valores?

- ¿Cuál es la dirección que están tomando estas transformaciones?

A la primera cuestión, la respuesta tendría que andar entre la tipología de los cambios sociales según si su impacto en la estructura social fuera revolucionario o no, o, dicho de otro modo, si subvierte radicalmente las instancias sociales provocando una crisis sociocultural, o tendremos que valorarlo más como una evolución lógica y superficial de aspectos parciales e institucionales de la sociedad. El siguiente interrogante nos plantea el clásico debate sobre la relación, influencia o determinismo entre la infraestructura y superestructura. $Y$ en último lugar, pero no menos interesante, es la apuesta por un ejercicio de prognosis social que sólo tiene sentido si responde a un profundo trabajo intelectual y empírico.

Las tres preguntas tienen una posible respuesta en los planteamientos de R. Inglehart, que culminan la tradición de los estudios de G. Almond y S. Verba sobre la «cultura cívica» (Almond y Verba, 1970; Almond, 1998) y trasladan al ámbito sociológico los trabajos de A. Maslow sobre la «jerarquía de necesidades» (Maslow, 1964, 1991). Asimismo, las tesis de Inglehart están fundamentadas empíricamente, ya que informan y concluyen sobre la base de los estudios y encuestas administrados a lo largo de dos décadas en todo el mundo sobre el cambio cultural de valores y de cultura política?

Sobre estos antecedentes teóricos como vectores más influyentes en su obra y sobre sus propios esfuerzos intelectuales y de investigación, Inglehart construye sus respuestas a las cuestiones anteriormente planteadas en torno al cambio social. A la pregunta de si nos encontramos o no ante una nueva fase de la cultura, el autor responde con el título de su obra más conocida y de mayor impacto, The Silent Revolution (La revolución silenciosa); dicho de otro modo, sí está ocurriendo una transformación estructural y cultural, pero ésta es progresiva, generacional y silenciosa. En segundo lugar, las vinculaciones complejas entre desarrollo socioeconómico y cambio sociocultural se han explicado tradicionalmente desde el marco teórico de la modernización que establecía entre ellos relaciones causales unidireccionales y deterministas.

Resuelve la cuestión sustentando el cambio cultural en el desarrollo tecnológico y económico de las sociedades occidentales, pero que, llegado a cierto punto, afirma Inglehart, la autonomía del ámbito cultural e incluso la influencia, que no determinismo, de éste sobre

7 La Encuesta Mundial de Valores (World Values Survey) se ha llevado a cabo en tres oleadas: 1981-1983, 1991-1993 y 19951996. En la actualidad se está realizando la cuarta. 
las estructuras socioeconómicas. Por último, responde a la tercera cuestión afirmando que las sociedades contemporáneas caminan hacia una sociedad postmaterialista.

En primer lugar, esto significa que en el plano individual se está cambiando hacia valores cada vez más postmaterialistas, como la autoexpresión y calidad de vida, y hacia un aumento de habilidades que permiten la mayor participación en política. En segundo lugar, en el plano del sistema social, el incremento en los niveles de desarrollo económico, cultural y educativo está reforzando aún más el cambio cultural y de valores (Inglehart, 1991, 1998).

La revitalización de la sociedad civil y el cambio cultural y de valores han servido de doble marco para situar el tema objeto de nuestra investigación, y que no es otro que acercarse a la cultura política y a los valores de esa porción de la sociedad civil que representan las asociaciones de voluntariado, no lucrativas, no gubernamentales, o también llamadas solidarias.

\section{LOS NUEVOS MOVIMIENTOS SOCIALES Y LAS ENTIDADES DE VOLUNTARIADO}

Los autores fundamentales para desarrollar los dos pilares teóricos que se han expuesto hasta ahora han sido, por una parte, Cohen y Arato, siempre inspirados en Habermas, que han defendido una versión restringida y una valoración potencialmente positiva de la sociedad civil, y, por otra parte, Inglehart, que ha demostrado un cambio cultural en el aumento de las habilidades cognitivas y en una tendencia hacia valores postmaterialistas. Unos y otro concluyen y concretan en los nuevos movimientos sociales (NMS), por un lado, la dimensión dinámica y potencialmente positiva de la sociedad civil y, por otro lado, el reflejo motivacional y la consecuencia lógica del cambio cultural y de valores; cada uno ejemplifica sus tesis deteniéndose en el análisis pormenorizado del movimiento feminista, los primeros (Cohen, 1983, 1985; Cohen y Arato, 1994), y en el movimiento ecologista, el segundo (Inglehart, 1991, 1992).

Las consideraciones e importancia concedida a los nuevos movimientos sociales por parte de Cohen y Arato para señalar el sector más dinámico de la sociedad civil, y por Inglehart para ejemplificar y vincularlos al cambio cultural y a los valores postmaterialistas, nos obligan a acercarnos a su definición y caracterización general, aunque sea brevemente.

Se podría señalar un listado importante de aspectos donde, de una manera u otra, encontramos diferencias significativas entre los «viejos» movimientos sociales y los NMS y, por consiguiente, los rasgos definitorios de estos últimos. En síntesis, destacamos como carac- 
terística distintiva sociocultural el modelo de sociedad postindustrial donde surgen los NMS, y aspectos diferenciadores socioinstitucionales que presentan los modos de actuar no-convencionales y de organización menos centralizada e informal de los NMS.

Si aplicamos una por una las características de los NMS, podemos concluir que las organizaciones de voluntarios poseen todos los rasgos señalados y, por tanto, podemos situarlas perfectamente dentro de aquéllos, a pesar de las críticas que prefieren calificarlos como «mal sustituto del Estado», más bien «grupo de interés» o fruto de «motivaciones religiosas en declive» (ver nota 3 ).

El desarrollo y la explosión del voluntariado y de sus organizaciones son el resultado de la llegada de la sociedad postindustrial, en el sentido de que la coincidencia en su crecimiento ha estado acompañada de este nuevo marco y modelo social ${ }^{8}$. Aunque si bien es cierto que el voluntariado existió en otros momentos históricos del pasado, la generalización y la determinación del concepto son relativamente recientes ${ }^{9}$. El voluntariado moderno nace con el tiempo liberado en la sociedad de la información y del ocio, que se sustancia en una racionalidad que no es estrictamente económica (García Roca, 1994: 31).

Otra razón se refiere a la denominación aplicada a aquellos participantes en los NMS y que en bastantes ocasiones es la de voluntario, contraponiéndolo en muchos casos al de militante que está afiliado a movimientos sociales tradicionales. El voluntariado ha sido formalmente el denominador común sin el cual los NMS como ecologistas y pacifistas no hubieran podido desarrollar sus estrategias y prácticas (Falcón, 1997: 9).

En este sentido, el voluntario nace de una opción libre, como el militante, pero mientras que uno busca intereses particulares o generales, por mor de unos determinados valores e identidades, el otro persigue defender sus intereses, ideas y valores en el ámbito de la lucha política y la conquista del poder. Como señala J. R. Capella, la idea de mili-

\footnotetext{
8 Ana de Felipe y Lilo Rodríguez diferencian entre factores estructurales (mayor disponibilidad de tiempo libre, crisis del Estado de Bienestar, crisis de valores, desencanto con los partidos políticos y confianza en las asociaciones no-lucrativas) y coyunturales (tragedia de Ruanda, la Plataforma del 0,7\%...) para explicar la explosión de la solidaridad, el aumento del número de voluntarios y de la creación de nuevas organizaciones (De Felipe y Rodríguez de Rivas, 1995: 81-83).

9 La acción voluntaria ha sido una tarea presente a lo largo de la historia desde que el cristianismo o el Islam consideraban que ayudar a los demás era un medio para alcanzar la vida eterna o una obligación instada desde el libro sagrado. En la Edad Media la familia y el vecindario se mostraron a través de bacines, obras pías y gremios como el camino para dar respuesta a las situaciones de necesidad. La labor caritativa de carácter religioso pervivió a lo largo de los siglos de la edad moderna y contemporánea, hasta que en el siglo xIX y principios del xx el Estado provisory organizaciones civiles comenzaron a simultanearse con las prácticas de beneficencia de las mujeres burguesas, aristocráticas y piadosas. A pesar de esta larga tradición histórica de voluntariado y acción caritativa, el voluntariado social de hoy, dentro de su heterogeneidad, "es una auténtica novedad histórica, por ser una prestación gratuita y espontánea de servicios a los demás y aquellas otras prestaciones premodernas, más establecida por obligación y para corresponder recíprocamente a la comunidad» (Gutiérrez Resa, 1997: 22).
} 
tancia lleva aparejada otros conceptos como son la disciplina (autoimpuesta o impuesta desde fuera), la unidad, la jerarquía, la obediencia, el sacrificio y la entrega... Por esta razón, propone el modelo de trabajador voluntario, basado en la concepción de considerarse un asociado entre iguales, que pone en común con los demás su trabajo no retribuido, cuyo destinatario es la sociedad y cuya actividad es pública pero no estatal (cfr. Zubero, 1996: 218-219).

Aún más importante que algunas características y cuestiones terminológicas es la idea de que las organizaciones de voluntariado de bienestar social recogen, codifican y definitivamente institucionalizan la rica y compleja revolución de los NMS, completando e implementando el Estado de Bienestar keynesiano en la ciudadanía. Dicho de otro modo, el fermento utópico y desmercantilizador de los NMS de los años sesenta ha sido el motor básico para la posibilidad y la existencia de este sector voluntario, tanto en su reciente incremento como en su cambio de orientación (Alonso, 1996: 105, 1998).

\section{LOS VALORES POSTMATERIALISTAS Y LA CULTURA POLÍTICA DEL VOLUNTARIADO}

Según la Encuesta Mundial de Valores de 1995-96 (EMV), administrada a 6.920 personas, el $15 \%$ de la población española reconocía estar asociada a organizaciones caritativas; del cual, el $47 \%$ se consideraba, además, miembro activo en dichas asociaciones. Esta pertenencia relativamente alta por parte de los españoles y esta baja implicación activa de los miembros en comparación con el resto de asociaciones nos lleva a concluir, en términos del profesor Ruiz Olabuénaga, que estamos frente a una organización de «éxito ritual»: es decir, que tiene gran capacidad para favorecer la afiliación de las personas, pero poca para la implicación directa en sus actividades (Ruiz Olabuénaga, 1994).

El perfil sociodemográfico del colectivo de miembros de asociaciones caritativas que predomina apunta hacia una mujer; adulta o madura; casada; de ingresos, clase social y estudios medios-altos; con alto grado de religiosidad; ideología de centro-derecha y derecha sobre-representada; y valores más postmaterialistas que materialistas (ver tabla 1).

El perfil descrito en el párrafo anterior se acentúa si seleccionamos sólo a los miembros activos, que, como se puede observar, son aún más mayoritariamente mujeres (58\%), adultos $(37 \%)$, casados $(60 \%)$, estudios altos $(34 \%)$ y religiosos $(79 \%)$, por un lado, y quedan sobre-representados en los ingresos medios-altos, en la clase social media-alta, en la ideología de centro-derecha y derecha, y en valores mixtos y postmaterialistas, por otro lado. 
TABLA 1

Perfil sociodemográfico de los miembros de organizaciones caritativas

\begin{tabular}{|c|c|c|c|c|}
\hline & & Activo & No activo & No miembro \\
\hline \multirow[t]{2}{*}{ Sexo } & Hombre & 42 & 43 & 49 \\
\hline & Mujer & 58 & 57 & 51 \\
\hline \multirow[t]{4}{*}{ Edad } & Joven & 25 & 29 & 33 \\
\hline & Adulto & 37 & 35 & 33 \\
\hline & Maduro & 23 & 21 & 18 \\
\hline & Mayor & 15 & 15 & 15 \\
\hline \multirow[t]{4}{*}{ Estudios } & Muy bajo & 14 & 18 & 26 \\
\hline & Bajo & 25 & 25 & 28 \\
\hline & Medio & 27 & 29 & 26 \\
\hline & Alto & 34 & 28 & 20 \\
\hline \multirow[t]{4}{*}{ Clase social } & Alta & 28 & 22 & 15 \\
\hline & Media & 24 & 26 & 25 \\
\hline & Trabajadora & 46 & 48 & 56 \\
\hline & Baja & 2 & 4 & 5 \\
\hline \multirow[t]{2}{*}{ Religiosidad } & Religioso & 79 & 72 & 70 \\
\hline & No-religioso & 21 & 28 & 30 \\
\hline \multirow[t]{5}{*}{ Ideología } & Izquierda & 10 & 14 & 16 \\
\hline & Centro-izquierda & 28 & 32 & 32 \\
\hline & Centro & 39 & 33 & 37 \\
\hline & Centro-derecha & 16 & 15 & 11 \\
\hline & Derecha & 6 & 6 & 4 \\
\hline \multirow[t]{3}{*}{ Materialista/postmaterialista } & Materialista & 15 & 17 & 22 \\
\hline & Mixto & 55 & 52 & 54 \\
\hline & Postmaterialista & 30 & 31 & 24 \\
\hline
\end{tabular}

FUENTE:

Elaboración propia (ASEP, 1999).

Del análisis detenido del perfil de los miembros activos de asociaciones caritativas se puede observar que una gran parte de las variables se asemejan en su distribución y porcentaje a los resultados de las organizaciones de la Iglesia. Esta percepción se confirma cuando destaca sobremanera el número de personas que son miembros de una y otra organización. Así, casi el $80 \%$ de los que afirman pertenecer a organizaciones caritativas también lo están en movimientos de la Iglesia. 
Dada la importancia de las variables de religiosidad y la escala de postmaterialismo, hemos llevado a cabo un análisis de segmentación colocando como variable dependiente ser miembro activo, miembro o no miembro de organizaciones caritativas, y como variables independientes la religiosidad (ser religioso o no) y el postmaterialismo (ser materialista o postmaterialista). Así, por una parte, aparece como primera variable predictora el postmaterialismo y en segundo lugar la religiosidad y, por otra parte, dentro de los postmaterialistas son los religiosos los más numerosos. Es decir, se concluye que de los cuatro posibles tipos éticos (religioso/materialista, religioso/postmaterialista, a-religioso/materialista y a-religioso/postmaterialista) resultantes de la combinación de las dos variables dicotomizadas y citadas más arriba, el más frecuente y sobre-representado es el religioso/postmaterialista entre los miembros activos de organizaciones caritativas. Además, si comparamos el peso que obtiene el tipo religioso/postmaterialista en el total de los encuestados (37\%) frente a los miembros activos de organizaciones caritativas (52\%) advertimos una importante y significativa diferencia (ver gráfico 2).

Tras analizar el perfil sociodemográfico y los valores postmaterialista, el estudio de la cultura política del voluntariado social nos ofrecerá otro aspecto poco estudiado desde el punto de vista empírico, ya que las investigaciones se han centrado especialmente en cuantificar y medir las dimensiones del fenómeno social que supone en los últimos años el voluntariado.

\section{GRÁFICO 2}

Comparación de los tipos éticos entre miembros activos de organizaciones caritativas y el total de los encuestados

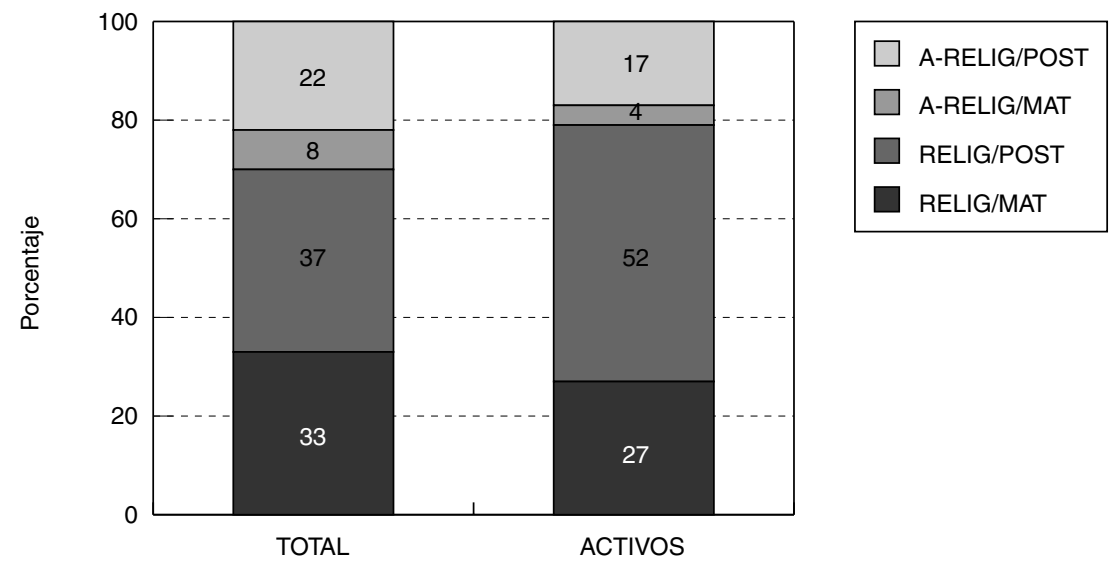

FUENTE:

Elaboración propia (ASEP, 1999). 
En este caso, tenemos la oportunidad de examinar a partir de los datos de la EMV en España algunas características de la cultura política y democrática del voluntariado social, puesto que, por una parte, el cuestionario recoge un número importante de preguntas relativas a estos temas, y la submuestra de aquellas personas que afirmaron pertenecer activamente o no a asociaciones caritativas es bastante significativa, 512 y 578 encuestados, respectivamente ${ }^{10}$. Con estas muestras vamos a trabajar, aunque no estén ponderadas para una inferencia a nivel estatal; el objetivo que pretendemos no es tanto obtener unos resultados para España como detectar algunas características globales o tendencias significativas en este colectivo perteneciente a organizaciones benéficas.

Las dimensiones de estudio de la cultura política aparecen de diferentes formas organizadas y de distintas maneras cuantificadas o enumeradas. Nuestra opción se reduce a señalar tres grandes dimensiones de la cultura política, habitualmente tratadas en la mayoría de los textos actuales y clásicos, y agrupar bajo ellas un subconjunto de preguntas aparecidas en el cuestionario de la EMV ${ }^{11}$.

La primera dimensión comprende el componente cognitivo o de preocupación, interés, actividad verbal que incita la opinión manifestada por los encuestados hacia la política.

La segunda dimensión se centra en el aspecto afectivo o de la confianza que provocan algunas de las instituciones más importantes de la vida política: el Gobierno de la nación, el Parlamento y los partidos políticos. Por último, la dimensión conductual o comportamental se centra en las llamadas acciones políticas no-convencionales como son, por ejemplo, la firma de una petición, boicot, manifestaciones legales, huelgas ilegales y ocupar edificios (ver tabla 2).

El análisis descriptivo de estas tres dimensiones a través de las medias resultantes en las once preguntas nos permite realizar diferentes consideraciones. Antes de comentar los resultados habría que hacer una salvedad sobre el tratamiento de los datos, ya que se han respetado las opciones de respuesta en el cálculo de la media; es decir, se han conserva-

\footnotetext{
10 Juan del Pino y Eduardo Bericat publicaron el informe de la última Encuesta Mundial de Valores en Andalucía, donde desarrollaron un amplio capítulo dedicado a la cultura política y democrática, que ha servido de inspiración para este apartado (Del Pino y Bericat, 1998).

11 M. ${ }^{a}$ Luz Morán y Jorge Benedicto citan un conjunto importante de dimensiones en el estudio de la cultura política española, muchas de las cuales van a ser tratadas en este apartado. Brevemente, el esquema empleado por los dos autores presenta cuatro bloques: Individuo-ciudadano como actor (valores sociales, creencias políticas básicas, experiencias de socialización política); vinculación ciudadano-política (implicación política personal, política y medios de comunicación, asociacionismo, participación política); imagen del sistema político (el sistema democrático, actores e instituciones políticas democráticas); los resultados de la acción institucional (juicio sobre la labor del Gobierno, Estado y bienestar social) (Morán y Benedicto, 1995: 33; Morán, 1999).
} 
do los valores de las diferentes variables. De esta manera, en la tabla 2 se ha señalado la horquilla de opciones desde 1 , que representa la mayor frecuencia, mejor valoración, la más alta importancia o ha hecho, hasta 3 ó 4, que indican la menor frecuencia, valoración, importancia o que nunca ha realizado en el caso de las acciones políticas.

Existen diferencias entre no miembros y miembros, tanto activos como los que no lo son, en la mayoría de los ítems; fundamentalmente, en los que se refieren a la dimensión cognitiva y conductual. Las diferencias que podemos encontrar entre miembros activos y no miembros en las acciones políticas no convencionales aparecen tan sólo en aquellos comportamientos que se sujetan a una cierta legalidad (firmas y manifestaciones), frente a aquellas otras acciones con connotaciones de ilegalidad, donde no encontramos ninguna variación. Mientras, en la dimensión afectiva, que trataba de la confianza en las instituciones políticas más importantes, las medias son prácticamente iguales en las tres categorías.

TABLA 2

Las dimensiones de la cultura política y la pertenencia a organizaciones caritativas

Importancia de la política (1-4)

Discute sobre política (1-3)

Interés por la política (1-4)

Confianza Gobierno nacional (1-4)

Confianza Parlamento (1-4)

Confianza partidos políticos (1-4)

Firmar una petición (1-3)

Boicot (1-3)

Manifestación (1-3)

Huelga ilegal (1-3)

Ocupar edificios (1-3)

\begin{tabular}{crrrrrrr} 
& Activo & \multicolumn{2}{c}{ No activo } & \multicolumn{2}{c}{ No miembro } & & Total \\
\hline $\bar{x}$ & $\mathrm{~s}_{\mathrm{x}}$ & $\overline{\mathrm{x}}$ & $\mathrm{s}_{\mathrm{x}}$ & $\overline{\mathrm{x}}$ & $\mathrm{s}_{\mathrm{x}}$ & $\overline{\mathrm{x}}$ & $\mathrm{s}_{\mathrm{x}}$ \\
\hline 2,8 & 1,0 & 2,9 & 0,9 & 3,1 & 0,9 & 3,1 & 0,9 \\
2,1 & 0,7 & 2,1 & 0,7 & 2,3 & 0,7 & 2,3 & 0,7 \\
2,7 & 1,1 & 2,8 & 1,0 & 3,0 & 1,0 & 3,0 & 1,0 \\
3,0 & 0,9 & 3,0 & 0,8 & 3,0 & 0,8 & 3,0 & 0,8 \\
2,9 & 0,8 & 2,9 & 0,8 & 2,9 & 0,8 & 2,9 & 0,8 \\
3,2 & 0,7 & 3,1 & 0,7 & 3,2 & 0,7 & 3,2 & 0,7 \\
1,8 & 0,8 & 1,8 & 0,8 & 2,0 & 0,8 & 1,9 & 0,8 \\
2,5 & 0,7 & 2,4 & 0,7 & 2,5 & 0,7 & 2,5 & 0,7 \\
1,7 & 0,8 & 1,9 & 0,8 & 1,9 & 0,8 & 1,9 & 0,8 \\
2,6 & 0,7 & 2,5 & 0,7 & 2,6 & 0,7 & 2,6 & 0,7 \\
2,7 & 0,6 & 2,6 & 0,6 & 2,7 & 0,5 & 2,7 & 0,5
\end{tabular}

FUENTE:

Elaboración propia (ASEP, 1999).

Con el objeto de hacer más visuales los resultados se han elaborado tres índices que resumen los valores medios obtenidos en cada una de las dimensiones. Tras la adición de los ítems correspondientes, se ha realizado una transformación lineal de las puntuaciones obtenidas para cada dimensión con valores de 0 a 10, donde 0 sería el tanteo más bajo y 10 el mayor. El gráfico logrado nos facilita la comparación de cada una de las dimensiones, 
detectando de nuevo, y en primer lugar, el aumento progresivo en las dimensiones cognitiva y conductual de las categorías de no miembro a miembro no activo y activo (ver gráfico 3).

GRÁFICO 3

Índices de las dimensiones en la cultura política y organizaciones caritativas
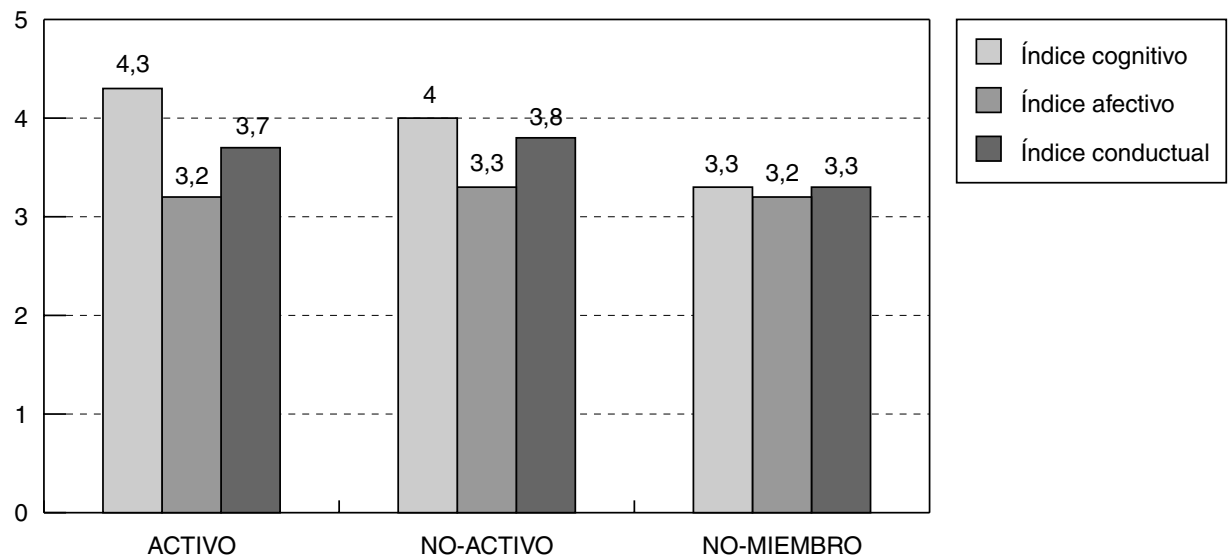

FUENTE:

Elaboración propia (ASEP, 1999).

En segundo lugar, existen diferencias entre los distintos índices sólo entre aquellos que pertenecen a asociaciones, comportándose del siguiente modo: destaca el índice cognitivo, siguiéndole el conductual, para relegarse en última posición el afectivo. En tercer lugar, centrados en las dimensiones cognitiva y conductual que ofrecen un comportamiento distinto entre los miembros de organizaciones caritativas y los que no lo son, observamos que las diferencias entre ellos aumentan.

La conclusión más relevante que podemos destacar del análisis de la cultura política a través de estas tres dimensiones es que la participación en asociaciones benéficas o caritativas aumenta los niveles de preocupación por la política (dimensión cognitiva) y participación en acciones políticas no-convencionales (dimensión conductual), pero no en una mayor valoración o confianza en las instituciones políticas (dimensión afectiva). Este rasgo de la cultura política de los miembros de organizaciones caritativas se completa con el aumento de la incongruencia entre los índices cognitivo y conductual. 
¿Cómo cabe interpretar dichos resultados? ¿Cómo es posible que a personas asociadas, movilizadas cognitivamente por la política, y activas en acciones políticamente no convencionales, no les acompañe una mejor valoración de la democracia y una mayor confianza en las instituciones políticas que la sustentan? Otro interrogante que queda en el aire tras el análisis de la cultura política es cómo explicar o comprender el aumento del diferencial entre la dimensión cognitiva y conductual entre los miembros activos de las organizaciones benéficas o caritativas.

\section{LOS VALORES Y LOS TRES SECTORES: LAS ENTIDADES DE VOLUNTARIADO SOCIAL Y LA SOLIDARIDAD}

El último de los análisis de datos realizados para conocer los valores de las entidades de voluntariado ha sido el fruto de la elaboración de una encuesta que se llevó a cabo expresamente en Almería, con el objeto de intentar probar dos hipótesis. Por una parte, identificar los tres reinos, sectores o espacios sociales pertenecientes al Estado, Mercado y Tercer Sector; y, por otra parte, pretendemos mostrar cuáles son los fines de cada uno de ellos siguiendo la tríada Poder, Dinero y Solidaridad.

La Encuesta Sociológica en Almería (ESA 99) se realizó en el término municipal de la capital, a mayores de 18 años y con una muestra total de 413 entrevistados. El cuestionario de este estudio contiene una batería de ítems donde se interroga sobre la valoración y los fines asociados a seis instituciones de Almería: Ayuntamiento, Diputación, Pryca, Alcampo, Cáritas y Almería Acoge.

Los resultados obtenidos nos confirman una vez más, junto con otras encuestas, que las instituciones de carácter caritativo o de voluntariado social tienen una valoración mucho más alta que otras organizaciones por parte de la opinión pública. En segundo lugar, a través del análisis factorial nos ha permitido concluir que los encuestados han identificado tres factores que agrupan las seis instituciones del siguiente modo: en el primer componente aparecen Ayuntamiento y Diputación; en el segundo, Alcampo y Pryca; y en el tercero, Almería Acoge y Cáritas, los cuales podrían cuadrar perfectamente con el rótulo de Estado, mercado y sociedad civil.

El último resultado a destacar ha sido demostrar la vinculación de las tres esferas o sectores a otros tantos valores o fines, tal y como los define Habermas, que afirma que el poder es el objetivo principal del Estado, el dinero del Mercado y la solidaridad de la sociedad civil ${ }^{12}$. De

12 En la Teoría de la Acción Comunicativa, Habermas distingue entre sistema y mundo de la vida (lebenswelt): el primero lo divide en los subsistemas económico y político, que persiguen el dinero y el poder, respectivamente, como única motivación 
este modo, interrogamos a los entrevistados sobre cuál era el fin de cada una de las seis organizaciones, ofreciendo como opciones de respuesta precisamente los tres fines mencionados por el sociólogo alemán. Así, la mayoría de los que respondieron a la encuesta señalaron como el objetivo principal del Ayuntamiento y Diputación el poder, 52 y $51 \%$, respectivamente. Casi la totalidad de las respuestas señalaron el dinero como la meta pretendida por Alcampo y Pryca (89\% en ambos casos); y también encontramos un amplísimo acuerdo en designar la solidaridad como el fin de Cáritas y Almería Acoge (93 y 92\% para cada una de las organizaciones de voluntariado social) (ver gráfico 4).

Los resultados confirman de manera alentadora las tesis expuestas por la Teoría de las Tres esferas (Habermas, 1987), de los Tres reinos (Cohen y Arato, 1994) o de los Tres sectores (Donati, 1997); la opinión pública identifica clara y distintamente los fines perseguidos por las instituciones que corresponden a los tres sectores.

Tal vez, la única nota discordante sea la importancia concedida al dinero como finalidad para las instituciones correspondientes al Estado o primer sector (Ayuntamiento y Diputación). Habría que interpretar con criterios de ciudadano-contribuyente la decisión de aquellos que optaron por asociar dinero con Ayuntamiento, ya que esta organización tiene capacidad recaudatoria.

A pesar de los contundentes resultados, la realidad social obliga a matizar y ponderar las conclusiones, ya que una de las consecuencias de la globalización supone la imbricación y hasta confusión de los distintos reinos o sectores estudiados. Así, hallaremos implicaciones del Estado en la tarea de la promoción del voluntariado y de las organizaciones del Tercer Sector a través de leyes, planes y subvenciones ${ }^{13}$. Por su parte, el Mercado también ha ido progresivamente planteando una mayor relación y compromiso en el ámbito del Tercer Sector a través del principio affinity o afinidad que podemos encontrar en las campañas de marketing con causa, los fondos de inversión éticos o en las mismas tarjetas de crédito y consumo solidarias que vinculan una organización voluntaria con una entidad bancaria o empresa ${ }^{14}$.

de una razón y acción instrumental; el segundo es el ámbito de los principios normativos y de la acción comunicativa (Habermas, 1987: vol. II, cap. 6).

\footnotetext{
13 A veces, la apuesta del Estado o Administración no queda sólo en compromiso de apoyo más o menos directo a las organizaciones de voluntariado social, sino que en multitud de ocasiones asume un protagonismo al organizar, captar y formar él mismo a voluntarios para campamentos de verano (el Ayuntamiento de Almería lo lleva realizando varios años) para tareas medioambientales (Agencia del Medio Ambiente) o para acompañar a ancianos (Servicios Sociales de la Junta de Andalucía).

14 Ejemplos de tarjetas solidarias: Asociación contra el Cáncer-Central Hispano; Ayuda en Acción-Central Hispano; Medicus Mundi-Caja Laboral; Adena-Central Hispano; Unicef-Caja Madrid; Arcoiris-Hispamer.
} 
GRÁFICO 4

Fines de las instituciones almerienses

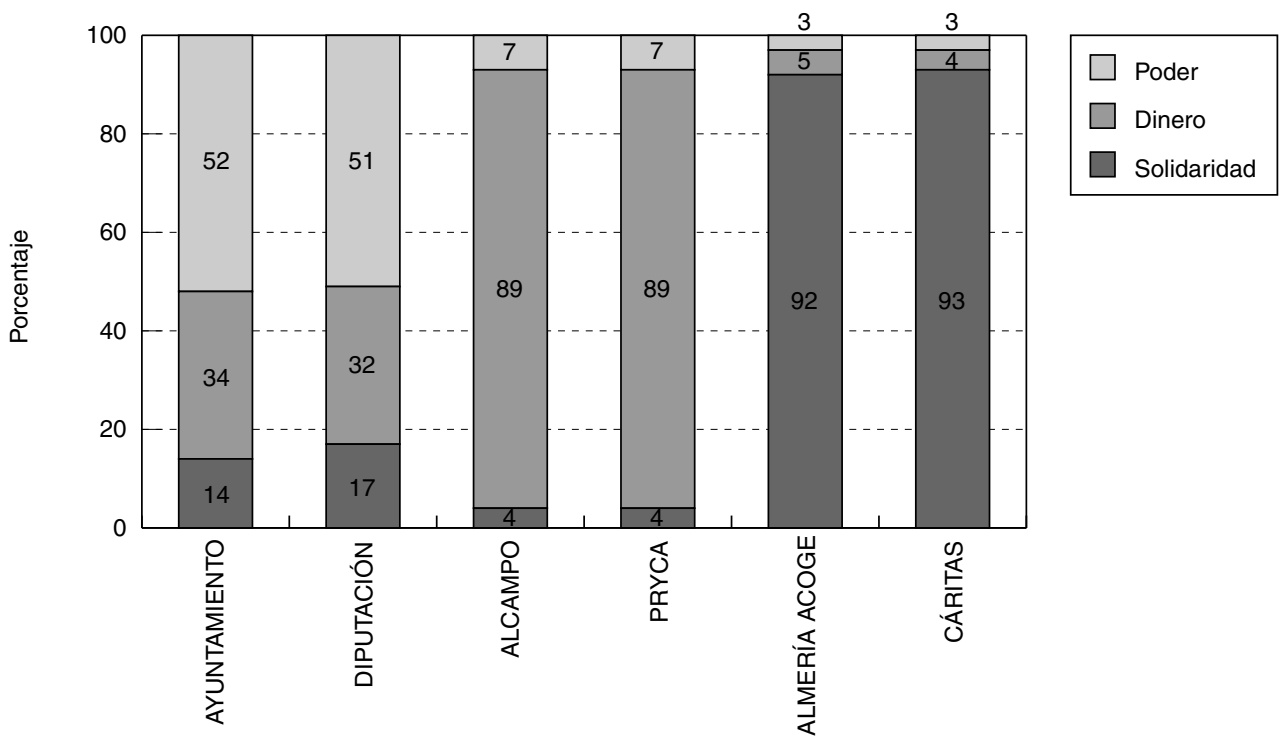

FUENTE:

Elaboración propia (ESA, 1999).

\section{CONCLUSIONES}

El concepto de sociedad civil en los últimos años se ha vuelto recurrente, entre otras razones por el aumento singular, constante y generalizado de un elenco de asociaciones que han adoptado los nombres de ONG, organizaciones no lucrativas, organizaciones de la sociedad civil, etc. El mayor número de recursos humanos y económicos en forma de tales instituciones, el protagonismo logrado en los cambios sociales contemporáneos (algunos de ellos trascendentales como la caída del muro y la vuelta al escenario del debate intelectual), hacen de la sociedad civil una realidad teórica y práctica ineludible en ciencias sociales.

Las interpretaciones de los últimos cambios sociales y culturales acaecidos en las sociedades complejas y occidentales han sido abordadas desde dos perspectivas bien distintas: la modernidad reflexiva y la postmodernidad. Estos dos planteamientos, más que oponerse, se complementan y enriquecen mutuamente, aunque habría que buscar una propuesta 
para la comprensión y explicación sintética del cambio social. Inglehart propone un modelo teórico para la interpretación del cambio cultural que señala como término del proceso progresivo, generacional y silencioso de transformaciones sociales, la sociedad postmaterialista. Esta sociedad se caracteriza por primar los valores de la auto-realización y de la libertad de expresión que implica, entre otros elementos, una repolitización de la población; dicho de otro modo, una movilización cognitiva o mayor preocupación por lo político.

Los nuevos movimientos sociales son, por una parte, la expresión del resurgir y renacimiento de la sociedad civil según Cohen y Arato, que toman como ejemplo las asociaciones feministas; $y$, por otra parte, aparecen como los mayores exponentes del cambio cultural propugnado por Inglehart, que escoge como paradigma las organizaciones ecologistas. Las organizaciones de voluntariado social y la nueva ola de ONG que trabajan en el ámbito de lo social surgidas a finales de los años ochenta y principios de los noventa, pertenecen a la tercera generación de los nuevos movimientos sociales al compartir gran parte de las características señaladas.

Las organizaciones de voluntariado social se sitúan en el marco del Tercer Sector (definido como la parte institucional y asociada de la sociedad civil) y se explican también desde las teorías sociológicas del Tercer Sector. Tanto las ONG de ámbito social como el Tercer Sector, y por ende la sociedad civil, están actualmente en un proceso de ebullición y en una tendencia al crecimiento generalizado que están empujando a un cambio cultural de carácter global.

Los miembros de organizaciones caritativas o benéficas comparten significativamente más valores postmaterialistas que el resto de la población; lo cual confirma la hipótesis de que las asociaciones voluntarias en el ámbito de lo social y el voluntariado social son parte de la punta de lanza del cambio cultural en los términos defendidos por Inglehart. Sin embargo, los miembros activos de estas organizaciones manifiestan ser también más religiosos que aquellos que no pertenecen, característica, en principio, contradictoria con los valores postmaterialistas.

Por tanto, las organizaciones caritativas están siendo el lugar de encuentro de una combinación singular entre valores religiosos y postmaterialistas; que están generando, a su vez, un tipo ético en el que se casan la caridad salvadora de origen cristiano con la solidaridad autoexpresiva de inspiración postmoderna; o el amor al prójimo basado en los mandamientos con la ayuda humanitaria fundamentada en los derechos humanos. Podremos afirmar, al fin, que una de las posibilidades de la postmodernidad, y que hemos tratado, era la vuelta hacia la premodernidad o síntesis de ambas; tesis que ayudaría a explicar este tipo ético reflejado y sobre-representado en el voluntariado social. Los miembros de asociaciones 
caritativas y benéficas que constituyen el voluntariado social poseen un conjunto de perfiles sociodemográficos, entre los que habría que destacar la alta participación de mujeres, la religiosidad, y otros rasgos del ámbito político como es el significativo grado de españolismo y la tendencia hacia la ideología de derechas frente al resto de organizaciones voluntarias.

En definitiva, el voluntariado social despliega un modelo de cultura política de fuerte carácter integrador, en el sentido de que su mayor participación y preocupación política no está orientada a cuestionar el statu quo ni el sistema político instituido. Evidentemente, su mayor movilización implica un enriquecimiento para la democracia y el sistema, aportándole contenidos.

Finalmente, en nuestro estudio sobre la población almeriense hemos podido confirmar la existencia de los tres sectores desde la valoración emitida por los encuestados sobre un conjunto de instituciones. Pero no sólo hemos constatado la claridad con la que se agrupan las organizaciones según el sector al que pertenezcan, sino que, además, hemos verificado la vinculación que posee cada una de las esferas sociales con los objetivos y metas definidos por Habermas: el poder es el fin perseguido por el Estado, el dinero por el mercado y la solidaridad por las organizaciones del Tercer Sector, entre las que sobresalen las asociaciones de voluntariado social.

\section{BIBLIOGRAFÍA}

ALEXANDER, J. C. (1998): "Civil Society I, II, III: Constructing an empirical concept from normative controversies and historical transformations», en J. Alexander (ed.), Real Civil Societies. Dilemmas of institutionalization, Beverly Hills: Sage.

ALMOND, G. A. (1998): «La historia intelectual del concepto de cultura cívica [traducción de un fragmento]», en R. del Águila, F. Vallespín y otros, La democracia en sus textos, Madrid: Alianza Editorial.

ALMOND, G. A., y VERBA, S. (1970): La cultura cívica, Madrid: Euroamérica.

ALONSO, L. E. (1996): «Nuevos movimientos sociales y asociacionismo», en G. Rodríguez Cabrero y J. Montserrat (dirs.), Las entidades voluntarias en España. Institucionalización, estructura económica y desarrollo asociativo, Madrid: Ministerio de Asuntos Sociales.

- (1998): «Los nuevos movimientos sociales en el umbral del año 2000», en Documentación Social, núm. 111, pp. 155-176.

ARATO, A. (1996): «Emergencia, declive y reconstrucción del concepto de sociedad civil. Pautas para análisis futuros», en Isegoría, núm. 13, pp. 5-17.

- (2000): Civil Society, Constitution, and Legitimacy, Maryland: Rowman \& Littlefield Publishers. 
BECK, U. (1998): ¿Qué es la globalización? Falacias del globalismo y respuestas a la globalización, Barcelona: Paídos.

COHEN, J. L. (1983): «Rethinking Social Movements», en Berkeley Journal of Sociology, vol. 28, pp. 97-113.

- (1985): «Strategy or identity: New Theoretical Paradigms and Contemporary Social Movements», en Social Research, vol. 52, núm. 4, pp. 663-716.

- (1998): «Interpreting the notion of Civil Society», en M. Walzer (ed.), Toward a Global Civil Society, Oxford: Berhahn Book.

COHEN, J. L., y ARATO, A. (1994): Civil Society and Political Theory, Cambrigde: MIT.

CORTINA, A. (1994): La ética de la sociedad civil, Madrid: Anaya.

- (1998): «Sociedad civil», en A. Cortina (dir.), 10 palabras claves en Filosofía Política, Navarra: Verbo Divino.

DARCY DE OLIVEIRA, M., y TANDON, R. (1996): «El surgimiento de una sociedad civil mundial», en Issues of Democracy (Spanish), julio, URL: http://usiahq.usis.usemb.se/journals/itdhr/0796/ijds/miguel.htm

DE FELIPE, A., y RODRÍGUEZ DE RIVAS, L. (1995): Guía de la solidaridad, Madrid: Temas de Hoy.

DEL PINO ARTACHO, J., y BERICAT, E. (1998): Valores sociales en la cultura andaluza. Encuesta Mundial de Valores. Andalucía 1996, Madrid: CIS.

DIAMOND, L. (2001): What Civil Society Can Do To Reform, Deepen, and Improve Democracy (Ponencia presentada en el Seminario "Civil Society, Social Capital, and Civic Engagement in Japan and the United States»).

DÍEZ NICOLÁS, J., e INGLEHART, R. (eds.) (1994): Tendencias mundiales del cambio en los valores sociales y políticos, Madrid: Fundesco.

DONATI, P. (1997): «El desarrollo de las organizaciones del Tercer Sector en el proceso de modernización y más allá», en REIS, núm. 79, pp. 113-141.

FALCÓN, E. (1997): Dimensiones políticas del voluntariado, Barcelona: Cristianisme i Justicia.

GARCÍA ROCA, J. (1994): Solidaridad y voluntariado, Santander: Sal Terrae.

GELLNER, E. (1996): Condiciones de la libertad. La sociedad civil y sus rivales, Barcelona: Paidós.

- (1998): Cultura, identidad y política, Barcelona: Gedisa.

GINER, S. (1996): Cartas sobre la democracia, Barcelona: Ariel.

- (1998): «Sociedad civil», en S. Giner, E. Lamo de Espinosa y C. Torres (eds.), Diccionario de Sociología, Madrid: Alianza Editorial.

GINER, S., y SARASA, S. (1995): «Altruismo cívico y política social», en Leviatán, núm. 61, pp. 67-98.

GUTIÉRREZ RESA, A. (1997): Acción social no gubernamental. Análisis y reflexiones sobre las organizaciones voluntarias, Valencia: Tirant lo Blanch.

HABERMAS, J. (1987): Teoría de la Acción Comunicativa II. Crítica de la razón funcionalista, Madrid: Taurus.

INGLEHART, R. (1991): El cambio cultural en las sociedades industriales avanzadas, Madrid: CIS.

- (1992): "Valores, ideología y movilización cognitiva en los nuevos movimientos sociales», en R. J. Dalton y M. Kuechler (comps.), Los nuevos movimientos sociales, Valencia: Edicions Alfons el Magnànim. 
INGLEHART, R. (1998): Modernización y posmodernización. El cambio cultural económico y político en 43 sociedades, Madrid: CIS.

KEANE, J. (1988): «Despotism and Democracy», en J. Keane (ed.), Civil Society and the State, new European perspectives, Londres: Verso.

- (1992): Democracia y sociedad civil, Madrid: Alianza Editorial.

MASLOW, A. K. (1964): Toward a psychology of being, Princeton: Van Nostrand.

- (1991): Motivación y personalidad, Madrid: Díaz de Santos.

MORÁN, M. ${ }^{a}$ L. (1999): «Los estudios de cultura política en España», en REIS, núm. 85, pp. 97-129.

MORÁN, M. ${ }^{a}$ L., y BENEDICTO, J. (1995): La cultura política de los españoles. Un ensayo de reinterpretación, Madrid: CIS.

PÉREZ-DÍAZ, V. (1987): El retorno de la sociedad civil, Madrid: Instituto de Estudios Económicos.

- (1993): La primacía de la sociedad civil. El proceso de formación de la España democrática, Madrid: Alianza Editorial.

— (1994): «La definición del espacio público europeo», en Claves de Razón Práctica, núm. 44, pp. 12-20.

— (1996): «Sociedad civil: Una interpretación y una trayectoria», en Isegoría, núm. 13, pp. 19-38.

- (1997): Esfera pública y la sociedad civil, Madrid: Taurus.

PUTNAM, R. D. (1996): «Bolear solo: El capital social de Estados Unidos en deterioro», en Issues of Democracy (Spanish), julio, URL: http://usinfo.state.gov/journals/itdhr/0796/ijds/putnam.htm

REVILLA BLANCO, M. (2002): Las ONG y la política, Madrid: ISTMO.

RUIZ OLABUÉNAGA, J. I. (1994): “Ocio y estilos de vida», en M. Juárez (dir.), V Informe sociológico sobre la situación social en España. Sociedad para todos en el año 2000, Madrid: Fundación FOESSA.

ZAIDI, S. A. (1998): «El fracaso de las organizaciones no gubernamentales (ONG's) y la necesidad de regresar al Estado», en Zona Abierta, núm. 82/83, pp. 75-85.

ZUBERO, I. (1996): Movimientos sociales y alternativas de sociedad, Madrid: Ediciones HOAC. 
ABSTRACT

The resurgence of the concept of civil society in the social sciences has one of its manifestations in the increase of the voluntary associations, in the last decades. This resurgence and increase in the associate participation of the citizen is currently, according to R. Inglehart, one of the culture shift indicators toward a post-materialistic society. In this search, we seek to define the concept of civil society following the pattern by J. L. Cohen and A. Arato, and to locate inside this, the voluntary associations and the Third Sector organizations. We also pursue to demonstrate, on one hand, that the active members in charitable and social organisations are part of the lance tip of the values change, and to show, on the other hand, the political and social culture of the voluntary organisations. 